\title{
Casimir and van der Waals force between two plates or a sphere (lens) above a plate made of real metals
}

\author{
G. L. Klimchitskaya, ${ }^{1, * \S}$ U. Mohideen, ${ }^{2, \dagger}$ and V. M. Mostepanenko ${ }^{1, \ddagger \S}$ \\ 1 Physics Department, Federal University of Paraíba, C.P.5008, \\ CEP 58059-970, João Pessoa, Pb-Brazil \\ 2 Department of Physics, University of California, Riverside, \\ California 92521
}

\begin{abstract}
The Casimir and van der Waals forces acting between two metallic plates or a sphere (lens) above a plate are calculated accounting for the finite conductivity of the metals. The simple formalism of surface modes is briefly presented which allows the possibility to obtain the generalization of Lifshitz results for the case of two semi-spaces covered by the thin layers. Additional clarifications of the regularization procedure provides the means to obtain reliable results not only for the force but also for the energy density. This, in turn, leads to the value of the force for the configuration of a sphere (lens) above a plate both of which are covered by additional layers. The Casimir interaction between $A l$ and $A u$ test bodies is recalculated using the optical tabulated data for the complex refractive index of these metals. The computations turn out to be in agreement with the perturbation theory up to the fourth order in relative penetration depth of electromagnetic zero point oscillations into the metal. The disagreements between the results recently presented in the literature are resolved. The Casimir force between $A l$ bodies covered by the thin $A u$ layers is computed and the possibility to neglect spatial dispersion effects is discussed as a function of the layer thickness. The van der Waals force is calculated including the transition region to the Casimir force. The pure non-retarded van der Waals force law between $A l$ and $A u$ bodies is shown to be restricted to a very narrow distance interval from $0.5 \mathrm{~nm}$ to $(2-4) \mathrm{nm}$. New, more exact, values of the Hamaker constant for $A l$ and $A u$ are determined.
\end{abstract}

12.20.Ds, 03.70.+k, 78.20.-e

\section{INTRODUCTION}

Recently considerable attention has been focussed on the van der Waals and Casimir forces acting between macroscopic bodies. As for the van der Waals force, interest in it has quickened owing to its application in atomic force microscopy (see, e.g., the monographs [1,2] and references therein). Interest in the Casimir force was rekindled after the new experiments [3, A] where it was measured more precisely in the case of metallic test bodies.

It is common knowledge that both forces are connected with the existence of zero point vacuum oscillations of the electromagnetic field [5.6]. For closely spaced macroscopic bodies the virtual photon emitted by an atom of one body reaches an atom of the second body during its lifetime. The correlated oscillations of the instantaneous induced dipole moments of those atoms give rise to the non-retarded van der Waals force. The Casimir force arises when the distance between two bodies is so large that the virtual photon emitted by an atom of one body cannot reach the second body during its lifetime. Nevertheless, the correlation of the quantized electromagnetic field in a vacuum state is not equal to zero at two points where the atoms belonging to different bodies are situated. Hence the non-zero correlations of the induced atomic dipole moments arise once more resulting in the Casimir force (which is also known as the retarded van der Waals force).

As is shown in $4,8,8$, the corrections to the Casimir force due to the finite conductivity of the metal and surface roughness play an important role in the proper interpretation of the measurement data. Temperature corrections

\footnotetext{
*On leave from North-West Polytechnical Institute, St.Petersburg, Russia. Electronic address: galina@GK1372.spb.edu

${ }^{\dagger}$ Electronic address: umar.mohideen@ucr.edu

${ }^{\ddagger}$ On leave from A.Friedmann Laboratory for Theoretical Physics, St.Petersburg, Russia. Electronic address: mostep@fisica.ufpb.br

${ }^{\S}$ Present Address: Institute for Theoretical Physics, Leipzig University, Augustusplatz 10/11, 04109, Leipzig, Germany.
} 
are negligible in the measurement range of [4, [, 8] (data of [3] do not support the presence of finite conductivity, surface roughness and temperature corrections which is in disagreement with the theoretically estimated values of these corrections [7] in the measurement range of [3]). In [4, ] the values of the finite conductivity corrections to the Casimir force were found by the use of perturbation expansion in relative penetration depth of electromagnetic zero point oscillations into the metal which starts from the general Lifshitz formula [9-11]. The parameter of this expansion is $\lambda_{p} /(2 \pi a)$, where $\lambda_{p}$ is the effective plasma frequency of the electrons, $a$ is the distance between interacting bodies. Note that the coefficient near the first order correction was obtained in 12,13, and near the second order one in [14 for the configuration of two plane parallel plates. In [3, 15] the results of [12, 13] and, correspondingly, [14] were modified for the configuration of a spherical lens above a plate. To do this the proximity force theorem [16] was applied. The coefficients to the third and fourth order terms of that expansion were first obtained in [17] for both configurations.

In applications to atomic force microscopy and the van der Waals force the Lifshitz formula and plasma model were used in 18,19] for different configurations of a tip above a plate. In [20,21, the density-functional theory along with the plasma model was used in the calculation of the van der Waals force. More complicated analytical representation for the dielectric permittivity (Drude model with approximate account of absorption bands) was used in [22] to calculate the van der Waals force between objects covered with a chromium layer with the Lifshitz formula.

The parameters of plasma and Drude models (plasma wavelength, electronic relaxation frequency) are not known very precisely. Due to this in 23 the attempt was undertaken to apply Lifshitz formalism numerically to gold, copper, and aluminum (see also [24]). The tabulated data for the frequency dependent complex refractive index of these metals were used together with the dispersion relation to calculate the values of dielectric permittivity on the imaginary frequency axis. Thereupon the Casimir force was calculated in [23] for configurations of two plates and a spherical lens above a plate in a distance range from $0.05 \mu \mathrm{m}$ to $2.5 \mu \mathrm{m}$. The same computation based on Lifshitz formalism and optical tabulated data for the dielectric permittivity was repeated in [25] in a distance range from $0.1 \mu \mathrm{m}$ to $10 \mu \mathrm{m}$. The two sets of results are in disagreement (see also 26]). Note that the higher-order perturbative calculations of [17] in their application range are in agreement with [25,26] but also disagree with [23, 24.

In this paper we present a brief derivation of the van der Waals and Casimir energy density and force between two parallel metallic plates or a plate and a sphere covered by the thin layers of another metal (the configuration used in the experiments [4. [8]). Two plates of sufficient thickness can be modelled by two semi-spaces with some gap between them. The case of multilayered plane walls was considered in [27]. In contrast to [27] where the removal of the infinities of the zero-point energy was not considered, we present explicitly the details of the regularization procedure and its physical justification. We next perform an independent computation using optical tabulated data for the frequency dependent complex refractive index of aluminum and gold with the goal to resolve the disagreement between earlier results. Our results turn out to be in agreement with [25,26] with a precision of computational error less than 1\%. Also the influence of the thin covering metallic layers onto the Casimir force is determined. The range of applicability and exceptions to using the bulk metal optical data for the dielectric permittivity of the thin metallic layers is discussed. For smaller distances the intermediate (transition) region between the Casimir and van der Waals forces is examined. It is shown that the transition region is very wide ranging from several nanometers to hundreds of nanometers. The pure van der Waals regime for aluminum and gold is restricted to separations in the interval from $0.5 \mathrm{~nm}$ till $(2-4) \mathrm{nm}$ only. The more exact values of the Hamaker constant for aluminum and gold are determined with the use of obtained computational data.

The paper is organized as follows. In Sec. II the general formalism is briefly presented giving the Casimir and van der Waals forces including the effect of covering layers on the surface of interacting bodies (two plates or a sphere above a plate). In Sec. III the influence of finite conductivity of the metal onto the Casimir force is reexamined. Sec. IV contains the calculation of the Casimir force between the aluminum surfaces covered by the thin gold layers. In Sec. V the van der Waals force is calculated in both configurations and the transition region to the Casimir is examined. Sec. VI contains determination of the Hamaker constant values for aluminum and gold. In Sec. VII we present conclusions and discussion, in particular, of possible applications of the obtained results in experimental investigations of the Casimir force and for obtaining stronger constraints on the constants of hypothetical long-range interactions.

\section{THE VAN DER WAALS AND CASIMIR FORCE BETWEEN LAYERED SURFACES: GENERAL FORMALISM}

We consider first two semi-spaces bounded by planes $(x, y)$ and filled with material having a frequency-dependent dielectric permittivity $\varepsilon_{2}(\omega)$. Let the planes bounding the semi-spaces be covered by layers of thickness $d$ made of the another material with a dielectric permittivity $\varepsilon_{1}(\omega)$. The magnetic permeabilities of both materials are taken to 
be equal to unity. The region of thickness $a$ between the layers (see Fig. 1) is empty space. According to [28,29] van der Waals and Casimir forces for the configuration under consideration can be found by consideration of the surface modes for which $\operatorname{div} \boldsymbol{E}=0, \operatorname{curl} \boldsymbol{E}=0$. The infinite zero-point energy of electromagnetic field, dependent on $a$ and $d$, is given by 27

$$
E(a, d)=\frac{1}{2} \hbar \sum_{\boldsymbol{k}, n}\left(\omega_{\boldsymbol{k}, n}^{(1)}+\omega_{\boldsymbol{k}, n}^{(2)}\right)
$$

Here $\omega_{\boldsymbol{k}, n}^{(1,2)}$ are the proper frequencies of the surface modes with two different polarizations of the electric field (parallel and perpendicular to the plane formed by $\boldsymbol{k}$ and $z$ axis correspondingly), $\boldsymbol{k}$ is the two-dimensional propagation vector in the $x y$-plane.

For the vacuum energy density per unit area of the bounding planes (which is also infinite) one obtains from (何)

$$
\mathcal{E}(a, d)=\frac{E(a, d)}{L^{2}}=\frac{\hbar}{4 \pi} \int_{0}^{\infty} k d k \sum_{n}\left(\omega_{\boldsymbol{k}, n}^{(1)}+\omega_{\boldsymbol{k}, n}^{(2)}\right)
$$

where $L$ is the side-length of bounding plane.

The frequencies of the surface modes $\omega_{\boldsymbol{k}, n}^{(1,2)}$ are found from the boundary conditions for the electric field and magnetic induction imposed at the points $z=-\frac{a}{2}-d,-\frac{a}{2}, \frac{a}{2}$, and $\frac{a}{2}+d$ [27]. These boundary conditions for each polarization lead to a system of eight linear homogeneous equations. The requirements that these equations have non-trivial solutions are

$$
\begin{aligned}
& \Delta^{(1)}\left(\omega_{\boldsymbol{k}, n}^{(1)}\right) \equiv e^{-R_{2}(a+2 d)}\left\{\left(r_{10}^{+} r_{12}^{+} e^{R_{1} d}-r_{10}^{-} r_{12}^{-} e^{-R_{1} d}\right)^{2} e^{R_{0} a}-\left(r_{10}^{-} r_{12}^{+} e^{R_{1} d}-r_{10}^{+} r_{12}^{-} e^{-R_{1} d}\right)^{2} e^{-R_{0} a}\right\}=0, \\
& \Delta^{(2)}\left(\omega_{\boldsymbol{k}, n}^{(2)}\right) \equiv e^{-R_{2}(a+2 d)}\left\{\left(q_{10}^{+} q_{12}^{+} e^{R_{1} d}-q_{10}^{-} q_{12}^{-} e^{-R_{1} d}\right)^{2} e^{R_{0} a}-\left(q_{10}^{-} q_{12}^{+} e^{R_{1} d}-q_{10}^{+} q_{12}^{-} e^{-R_{1} d}\right)^{2} e^{-R_{0} a}\right\}=0 .
\end{aligned}
$$

Here the following notations are introduced

$$
r_{\alpha \beta}^{ \pm}=R_{\alpha} \varepsilon_{\beta} \pm R_{\beta} \varepsilon_{\alpha}, \quad q_{\alpha \beta}^{ \pm}=R_{\alpha} \pm R_{\beta}, \quad R_{\alpha}^{2}=k^{2}-\varepsilon_{\alpha} \frac{\omega^{2}}{c^{2}}, \quad \varepsilon_{0}=1, \quad \alpha=0,1,2 .
$$

Note that to obtain Eqs. (3) we set the determinants of the linear system of equations equal to zero and do not perform any additional transformations. This is the reason why (3) does not coincide with the corresponding equations of [5,27] where some transformations were used which are not equivalent in the limit $|\omega| \rightarrow \infty$ (see below).

Summation in (2) over the solutions of (3) can be performed with the help of the argument principle which was applied for this purpose in 28]. According to this principle

$$
\sum_{n} \omega_{\boldsymbol{k}, n}^{(1,2)}=\frac{1}{2 \pi i}\left[\int_{i \infty}^{-i \infty} \omega d \ln \Delta^{(1,2)}(\omega)+\int_{C_{+}} \omega d \ln \Delta^{(1,2)}(\omega)\right]
$$

where $C_{+}$is a semicircle of infinite radius in the right one-half of the complex $\omega$-plane with a center at the origin. Notice that the functions $\Delta^{(1,2)}(\omega)$, defined in $(3)$, have no poles. For this reason the sum over their poles is absent from (5).

The second integral in the right-hand side of (5) is simply calculated with the natural supposition that

$$
\lim _{\omega \rightarrow \infty} \varepsilon_{\alpha}(\omega)=1, \quad \lim _{\omega \rightarrow \infty} \frac{d \varepsilon_{\alpha}(\omega)}{d \omega}=0
$$

along any radial direction in complex $\omega$-plane. The result is infinite, and does not depend on $a$ :

$$
\int_{C_{+}} \omega d \ln \Delta^{(1,2)}(\omega)=4 \int_{C_{+}} d \omega .
$$

Now we introduce a new variable $\xi=-i \omega$ in (5), (7). The result is 


$$
\sum_{n} \omega_{\boldsymbol{k}, n}^{(1,2)}=\frac{1}{2 \pi} \int_{\infty}^{-\infty} \xi d \ln \Delta^{(1,2)}(i \xi)+\frac{2}{\pi} \int_{C_{+}} d \xi
$$

where both contributions in the right-hand side diverge. To remove the divergences we use the regularization procedure which goes back to the original Casimir paper [30] (see also [6,28]). The idea of this procedure is that the regularized physical vacuum energy density vanishes for the infinitely separated interacting bodies. From Eqs. (3), (8) it follows

$$
\lim _{a \rightarrow \infty} \sum_{n} \omega_{\boldsymbol{k}, n}^{(1,2)}=\frac{1}{2 \pi} \int_{\infty}^{-\infty} \xi d \ln \Delta_{\infty}^{(1,2)}(i \xi)+\frac{2}{\pi} \int_{C_{+}} d \xi
$$

where the asymptotic behavior of $\Delta^{(1,2)}$ at $a \rightarrow \infty$ is given by

$$
\Delta_{\infty}^{(1)}=e^{\left(R_{0}-R_{2}\right) a-2 R_{2} d}\left(r_{10}^{+} r_{12}^{+} e^{R_{1} d}-r_{10}^{-} r_{12}^{-} e^{-R_{1} d}\right)^{2}, \quad \Delta_{\infty}^{(2)}=e^{\left(R_{0}-R_{2}\right) a-2 R_{2} d}\left(q_{10}^{+} q_{12}^{+} e^{R_{1} d}-q_{10}^{-} q_{12}^{-} e^{-R_{1} d}\right)^{2} .
$$

Now the regularized physical quantities are found with the help of (8)-(10)

$$
\left(\sum_{n} \omega_{\boldsymbol{k}, n}^{(1,2)}\right)_{r e g} \equiv \sum_{n} \omega_{\boldsymbol{k}, n}^{(1,2)}-\lim _{a \rightarrow \infty} \sum_{n} \omega_{\boldsymbol{k}, n}^{(1,2)}=\frac{1}{2 \pi} \int_{\infty}^{-\infty} \xi d \ln \frac{\Delta^{(1,2)}(i \xi)}{\Delta_{\infty}^{(1,2)}(i \xi)} .
$$

They can be transformed to a more convenient form with the help of integration by parts

$$
\left(\sum_{n} \omega_{\boldsymbol{k}, n}^{(1,2)}\right)_{r e g}=\frac{1}{2 \pi} \int_{-\infty}^{\infty} d \xi \ln \frac{\Delta^{(1,2)}(i \xi)}{\Delta_{\infty}^{(1,2)}(i \xi)}
$$

where the term outside the integral vanishes.

To obtain the physical, regularized Casimir energy density one should substitute the regularized quantities (12) into (2) instead of (8) with the result

$$
\mathcal{E}_{\text {reg }}(a, d)=\frac{\hbar}{4 \pi^{2}} \int_{0}^{\infty} k d k \int_{0}^{\infty} d \xi\left[\ln Q_{1}(i \xi)+\ln Q_{2}(i \xi)\right]
$$

where

$$
\begin{aligned}
& Q_{1}(i \xi) \equiv \frac{\Delta^{(1)}(i \xi)}{\Delta_{\infty}^{(1)}(i \xi)}=1-\left(\frac{r_{10}^{-} r_{12}^{+} e^{R_{1} d}-r_{10}^{+} r_{12}^{-} e^{-R_{1} d}}{r_{10}^{+} r_{12}^{+} e^{R_{1} d}-r_{10}^{-} r_{12}^{-} e^{-R_{1} d}}\right)^{2} e^{-2 R_{0} a} \\
& Q_{2}(i \xi) \equiv \frac{\Delta^{(2)}(i \xi)}{\Delta_{\infty}^{(2)}(i \xi)}=1-\left(\frac{q_{10}^{-} q_{12}^{+} e^{R_{1} d}-q_{10}^{+} q_{12}^{-} e^{-R_{1} d}}{q_{10}^{+} q_{12}^{+} e^{R_{1} d}-q_{10}^{-} q_{12}^{-} e^{-R_{1} d}}\right)^{2} e^{-2 R_{0} a}
\end{aligned}
$$

In (13) $Q_{1,2}$ are even functions of $\xi$ has been taken into account.

For the convenience of numerical calculations below we introduce the new variable $p$ instead of $k$ defined by

$$
k^{2}=\frac{\xi^{2}}{c^{2}}\left(p^{2}-1\right) .
$$

In terms of $p, \xi$ the Casimir energy density (13) takes the form

$$
\mathcal{E}_{\text {reg }}(a, d)=\frac{\hbar}{4 \pi^{2} c^{2}} \int_{1}^{\infty} p d p \int_{0}^{\infty} \xi^{2} d \xi\left[\ln Q_{1}(i \xi)+\ln Q_{2}(i \xi)\right]
$$

where a more detailed representation for the functions $Q_{1,2}$ from (14) is 


$$
\begin{aligned}
& Q_{1}(i \xi)=1-\left[\frac{\left(K_{1}-\varepsilon_{1} p\right)\left(\varepsilon_{2} K_{1}+\varepsilon_{1} K_{2}\right)-\left(K_{1}+\varepsilon_{1} p\right)\left(\varepsilon_{2} K_{1}-\varepsilon_{1} K_{2}\right) e^{-2 \frac{\xi}{c} K_{1} d}}{\left(K_{1}+\varepsilon_{1} p\right)\left(\varepsilon_{2} K_{1}+\varepsilon_{1} K_{2}\right)-\left(K_{1}-\varepsilon_{1} p\right)\left(\varepsilon_{2} K_{1}-\varepsilon_{1} K_{2}\right) e^{-2 \frac{\xi}{c} K_{1} d}}\right]^{2} e^{-2 \frac{\xi}{c} p a}, \\
& Q_{2}(i \xi)=1-\left[\frac{\left(K_{1}-p\right)\left(K_{1}+K_{2}\right)-\left(K_{1}+p\right)\left(K_{1}-K_{2}\right) e^{-2 \frac{\xi}{c} K_{1} d}}{\left(K_{1}+p\right)\left(K_{1}+K_{2}\right)-\left(K_{1}-p\right)\left(K_{1}-K_{2}\right) e^{-2 \frac{\xi}{c} K_{1} d}}\right]^{2} e^{-2 \frac{\xi}{c} p a} .
\end{aligned}
$$

Here all permittivities depend on $i \xi$ and

$$
K_{\alpha}=K_{\alpha}(i \xi) \equiv \sqrt{p^{2}-1+\varepsilon_{\alpha}(i \xi)}=\frac{c}{\xi} R_{\alpha}(i \xi), \quad \alpha=1,2 .
$$

For $\alpha=0$ one has $p=c R_{0} / \xi$ which is equivalent to 15 .

Notice that the expressions (13), (16) give us the finite values of the Casimir energy density which is in less common use than the force. Thus in 5 no finite expression for the energy density is presented for two semi-spaces. In 27] the omission of infinities is performed implicitly, namely instead of Eqs. (3) the result of their division by the terms containing $\exp \left(R_{0} a\right)$ was presented. The coefficient near $\exp \left(R_{0} a\right)$, however, turns into infinity on $C_{+}$. In other words the Eqs. (3) are divided by infinity. As a result the integral along $C_{+}$is equal to zero in [27] and the quantity (2) would seem to be finite. Fortunately, this implicit division is equivalent to the regularization procedure explicitly presented above. That is why the final results obtained in [27] are indeed correct. In 11 the energy density is not considered at all.

From (16) it is easy to obtain the Casimir force per unit area acting between semi-spaces covered with layers

$$
F_{s s}(a, d)=-\frac{\partial \mathcal{E}_{r e g}(a, d)}{\partial a}=-\frac{\hbar}{2 \pi^{2} c^{3}} \int_{1}^{\infty} p^{2} d p \int_{0}^{\infty} \xi^{3} d \xi\left[\frac{1-Q_{1}(i \xi)}{Q_{1}(i \xi)}+\frac{1-Q_{2}(i \xi)}{Q_{2}(i \xi)}\right] .
$$

This expression coincides with Lifshitz result [9-11] for the force per unit area between semi-spaces with a dielectric permittivity $\varepsilon_{2}$ if the covering layers are absent. To obtain this limiting case from (19) one should put $d=0$ and $\varepsilon_{1}=\varepsilon_{2}$

$$
F_{s s}(a)=-\frac{\hbar}{2 \pi^{2} c^{3}} \int_{1}^{\infty} p^{2} d p \int_{0}^{\infty} \xi^{3} d \xi\left\{\left[\left(\frac{K_{2}+\varepsilon_{2} p}{K_{2}-\varepsilon_{2} p}\right)^{2} e^{2 \frac{\xi}{c} p a}-1\right]^{-1}+\left[\left(\frac{K_{2}+p}{K_{2}-p}\right)^{2} e^{2 \frac{\xi}{c} p a}-1\right]^{-1}\right\} .
$$

The corresponding quantity for the energy density follows from (16)

$$
\mathcal{E}_{\text {reg }}(a)=\frac{\hbar}{4 \pi^{2} c^{2}} \int_{1}^{\infty} p d p \int_{0}^{\infty} \xi^{2} d \xi\left\{\ln \left[1-\left(\frac{K_{2}-\varepsilon_{2} p}{K_{2}+\varepsilon_{2} p}\right)^{2} e^{-2 \frac{\xi}{c} p a}\right]+\ln \left[1-\left(\frac{K_{2}-p}{K_{2}+p}\right)^{2} e^{-2 \frac{\xi}{c} p a}\right]\right\} .
$$

The other possibility to obtain the force between semi-spaces (but with a permittivity $\varepsilon_{1}$ ) is to consider limit $d \rightarrow \infty$ in (19). In this limit we obtain once more the results (20), (21) where $K_{2}, \varepsilon_{2}$ are replaced by $K_{1}, \varepsilon_{1}$. Note also that we do not take into account the effect of non-zero point temperature which is negligible for $a \ll \hbar c / T$.

The independent expression for the physical energy density is especially important because it allows the possibility to obtain approximate value of the force for the configuration of a sphere (or a spherical lens) above a semi-space. Both bodies can be covered by the layers of another material. According to the proximity force theorem this force is

$$
F_{s l}(a, d)=2 \pi R \mathcal{E}_{r e g}(a, d)=\frac{\hbar R}{2 \pi c^{2}} \int_{1}^{\infty} p d p \int_{0}^{\infty} \xi^{2} d \xi\left[\ln Q_{1}(i \xi)+\ln Q_{2}(i \xi)\right],
$$

where $R$ is the sphere radius, $Q_{1,2}$ are defined in (17). In the absence of layers $\mathcal{E}_{\text {reg }}(a, d)$ should be substituted by $\mathcal{E}_{\text {reg }}(a)$ from 21$)$.

Although the expression (22) is not exact it allows the possibility to calculate the force with a very high accuracy. As was shown in [6] (see also [31,32]) the proximity force theorem is equivalent to additive summation of interatomic van der Waals and Casimir force potentials with a subsequent normalization of the interaction constant. As was shown in [33] the accuracy of such method is very high (the relative error of the obtained results is less than $0.01 \%$ ) if the configuration corresponds closely with two semi-spaces which is the case for a sphere (lens) of a large radius $R \gg a$ above a semi-space.

In the following Sections the above general results will be used for computation of the Casimir and van der Waals forces acting between real metals. 


\section{THE INFLUENCE OF FINITE CONDUCTIVITY ON THE CASIMIR FORCE}

Let us first consider semi-spaces made of aluminum or gold. Aluminum covered interacting bodies (a plate and a lens) were used in the experiments [4, 8] because of its high reflectivity for wavelengths (plate-sphere separations) larger than $100 \mathrm{~nm}$. The thickness of $A l$ covering layer was $300 \mathrm{~nm}$. It is significantly greater than the effective penetration depth of the electromagnetic zero point oscillations into $A l$ which is $\delta_{0}=\lambda_{p} /(2 \pi) \approx 17$ nm (see Introduction). That is why $A l$ layer can be considered as infinitely thick and modelled by a semi-space. In the experiment [3] the test bodies were covered by a $500 \mathrm{~nm} A u$ layer which also can be considered as infinitely thick. In [4] and [8] $A l$ surfaces were covered, respectively, by $d<20 \mathrm{~nm}$ and $d=8 \mathrm{~nm}$ sputtered $A u / P d$ layers to reduce the oxidation processes in $A l$ and the effect of any associated electrostatic charges. The influence of such additional thin layers on the Casimir force is discussed in Sec. IV.

The values of the force per unit area for the configuration of two semi-spaces and the force for a sphere above a semi-space are given by Eq. (20) and Eqs. (21), (22). For the distance $a$ much larger than the characteristic wavelength of absorption spectra of the semi-space material $\lambda_{0}$ Eqs. (20), (21) lead [1] to the following results in the case of ideal metal $\left(\varepsilon_{2} \rightarrow \infty\right)$

$$
F_{s s}^{(0)}(a)=-\frac{\pi^{2}}{240} \frac{\hbar c}{a^{4}}, \quad F_{s l}^{(0)}(a)=-\frac{\pi^{3}}{360} R \frac{\hbar c}{a^{3}} .
$$

To calculate numerically the corrections to (23) due to the finite conductivity of a metal we use the tabulated data for the complex index of refraction $n+i k$ as a function of frequency [34]. The values of dielectric permittivity along the imaginary axes can be expressed through $\operatorname{Im} \varepsilon(\omega)=2 n k$ with the help of dispersion relation [11]

$$
\varepsilon(i \xi)=1+\frac{2}{\pi} \int_{0}^{\infty} \frac{\omega \operatorname{Im} \varepsilon(\omega)}{\omega^{2}+\xi^{2}} d \omega .
$$

Here the complete tabulated refractive indices extending from $0.04 \mathrm{eV}$ to $10000 \mathrm{eV}$ for $\mathrm{Al}$ and from $0.1 \mathrm{eV}$ to $10000 \mathrm{eV}$ for $\mathrm{Au}$ from 34 are used to calculate $\operatorname{Im} \varepsilon(\omega)$. For frequencies below $0.04 \mathrm{eV}$ in the case of $\mathrm{Al}$ and below $0.1 \mathrm{eV}$ in the case of $\mathrm{Au}$, the table values of 34 can be extrapolated using the free electron Drude model. In this case, the dielectric permittivity along the imaginary axis is represented as:

$$
\varepsilon_{\alpha}(i \xi)=1+\frac{\omega_{p \alpha}^{2}}{\xi(\xi+\gamma)}
$$

where $\omega_{p \alpha}=(2 \pi c) / \lambda_{p \alpha}$ is the plasma frequency and $\gamma$ is the relaxation frequency. $\mathrm{A} \omega_{p}=12.5 \mathrm{eV}$ and $\gamma=0.063 \mathrm{eV}$ was used for the case of $\mathrm{Al}$ based on the last results in Table XI on p.394 of [34]. In the case of Au the analysis is not as straightforward, but proceeding in the manner outlined in [25] we obtain $\omega_{p}=9.0 \mathrm{eV}$ and $\gamma=0.035 \mathrm{eV}$. While the values of $\omega_{p}$ and $\gamma$ based on the optical data of various sources might differ slightly we have found that the resulting numerically computed Casimir forces to differ by less than $1 \%$. In fact, if for Al metal, a $\omega_{p}=11.5 \mathrm{eV}$ and $\gamma=0.05 \mathrm{eV}$ as in 25] is used, the differences are extremely small. Of the values tabulated below, only the value of the force in the case of a sphere and a semi-space at $0.5 \mu \mathrm{m}$ separation is increased by $0.1 \%$ which on round-off to the second significant figure leads to an increase of $1 \%$. The results of numerical integration by Eq. (24) for $A l$ (solid curve) and $A u$ (dashed curve) are presented in Fig.2 in a logarithmic scale. As is seen from Fig 2 the dielectric permittivity along the imaginary axis decreases monotonically with increasing frequency (in distinction to $\operatorname{Im} \varepsilon(\omega)$ which possesses peaks corresponding to inter-band absorption).

The obtained values of the dielectric permittivity along the imaginary axis were substituted into Eqs. (20) and (22) (with account of (21)) to calculate the Casimir force acting between real metals in configurations of two semi-spaces (ss) and a sphere (lens) above a semi-space (sl). Numerical integration was done from an upper limit of $10^{4} \mathrm{eV}$ to a lower limit of $10^{-6} \mathrm{eV}$. Changes in the upper limit or lower limit by a factor of 10 lead to changes of less than $0.25 \%$ in the Casimir force. If the trapezoidal rule is used in the numerical integration of Eqs. (24) the corresponding Casimir force decreases by a factor less than $0.5 \%$. The results are presented in Fig. 3(a) (two semi-spaces) and in Fig. 3(b) for a sphere above a semi-space by the solid lines 1 (material of the test bodies is aluminum) and 2 (material is gold). In the vertical axis the relative force $F_{s s} / F_{s s}^{(0)}$ is plotted in Fig. 3(a) and $F_{s l} / F_{s l}^{(0)}$ in Fig. 3(b). These quantities provide a sense of the correction factors to the Casimir force due to the effect of finite conductivity. In the horizontal axis the space separation is plotted in the range $0.1-1 \mu \mathrm{m}$. We do not present the results for larger distances because the temperature corrections to the Casimir force become significant. At room temperature the temperature corrections contribute only $2.6 \%$ of $F_{s l}^{(0)}$ at $a=1 \mu \mathrm{m}$, but at $a=3 \mu \mathrm{m}$ they contribute $47 \%$ of $F_{s l}^{(0)}$, and at $a=5 \mu \mathrm{m}-129 \%$ of 
$F_{s l}^{(0)}$ 35]. It is seen that the relative force for $A l$ is larger than for $A u$ at the same separations as it should be because of better reflectivity properties of $A l$.

It is interesting to compare the obtained results with those of Refs. [23,24] and [25,26] where the similar computations were performed (in [25,26] the analytical expressions equivalent to Eqs. (20) and (21) were used, in [23,24], however, the energy density between plates was obtained by a numerical integration of the force which can lead to some additional error). All the results for the several values of distance between the test bodies are presented in the Table 1.

TABLE I. The correction factor to the Casimir force due to the finite conductivity of the metal by the results of different authors and the present paper in configurations of two semi-spaces (ss) and a sphere (lens) above a semi-space (sl).

\begin{tabular}{|c|c|c|c|c|c|c|}
\hline \multirow{2}{*}{$\begin{array}{c}\text { Test } \\
\text { bodies }\end{array}$} & \multirow[t]{2}{*}{ Metal } & \multirow{2}{*}{$\begin{array}{c}a \\
(\mu \mathrm{m})\end{array}$} & \multicolumn{4}{|c|}{$F / F^{(0)}$} \\
\hline & & & 23,24 & $\begin{array}{c}\text { Computation } \\
\text { [25,26 }\end{array}$ & This paper & $\begin{array}{c}\text { Perturbation } \\
\text { theory } \sqrt{17}\end{array}$ \\
\hline SS & $\overline{A l}$ & 0.1 & 0.557 & 0.55 & 0.55 & 0.56 \\
\hline sl & $A l$ & 0.1 & 0.651 & 0.63 & 0.62 & 0.61 \\
\hline sS & $A u$ & 0.1 & - & 0.48 & 0.49 & 0.62 \\
\hline sl & $A u$ & 0.1 & - & 0.55 & 0.56 & 0.60 \\
\hline ss & $A l$ & 0.5 & - & 0.85 & 0.84 & 0.84 \\
\hline sl & $A l$ & 0.5 & - & 0.88 & 0.87 & 0.88 \\
\hline ss & $A u$ & 0.5 & 0.657 & 0.81 & 0.81 & 0.81 \\
\hline sl & $A u$ & 0.5 & 0.719 & 0.85 & 0.85 & 0.85 \\
\hline sl & $A u$ & 0.6 & 0.78 & 0.87 & 0.87 & 0.87 \\
\hline ss & $A l$ & 3 & - & 0.96 & 0.96 & 0.97 \\
\hline sl & $A l$ & 3 & - & 0.97 & 0.97 & 0.98 \\
\hline ss & $A u$ & 3 & - & 0.96 & 0.95 & 0.96 \\
\hline sl & $A u$ & 3 & - & 0.97 & 0.96 & 0.97 \\
\hline
\end{tabular}

As is seen from Table 1, our calculational results (column 6) are in agreement with [25,26 (column 5) up to 0.01. At the same time the results of 23,24] (column 4) for $A u$ are in disagreement with both 25,26] and this paper. The results for $A l$ are presented in 23 at $a=0.1 \mu \mathrm{m}$ only. Note that the results at $a=3 \mu \mathrm{m}$ (the last four lines of the Table 1) are valid only at zero temperature. They do not take into account temperature corrections which are significant for such separation. Also the results of [23:24] for $C u$ covered bodies are in disagreement with [25]26]. We do not consider $C u$ here because the outer surfaces in the recent experiments were covered by the thick layers of $A u$ [3] and $A l$ [4, 8]. The hypothesis of [24] that the $A u$ film of $0.5 \mu \mathrm{m}$ thickness could significantly diffuse into the $C u$ layer of the same thickness at room temperatures seems unlikely. In any case it is not needed because the dielectric permittivity of $A u$ and $C u$ along the imaginary axis is almost the same [17,25,26] and, consequently, will also lead to the same Casimir force.

The computational results obtained here are in good agreement with analytical perturbation expansions of the Casimir force in powers of relative penetration depth $\delta_{0}=\lambda_{p} /(2 \pi)$ of the electromagnetic zero point oscillations into the metal. Representation (25) with $\gamma=0$ is applicable for the wavelengths (space separations) larger than $\lambda_{p \alpha}$ (the corrections due to relaxation processes are small for the distances $a \leq 5 \mu \mathrm{m}$ ). It can be substituted into Eqs. (20), (21) to get the perturbation expansion. According to the results of Ref. [17] the relative Casimir force with finite conductivity corrections up to the 4 th power is

$$
\frac{F_{s s}(a)}{F_{s s}^{(0)}(a)}=1-\frac{16}{3} \frac{\delta_{0}}{a}+24 \frac{\delta_{0}^{2}}{a^{2}}-\frac{640}{7}\left(1-\frac{\pi^{2}}{210}\right) \frac{\delta_{0}^{3}}{a^{3}}+\frac{2800}{9}\left(1-\frac{163 \pi^{2}}{7350}\right) \frac{\delta_{0}^{4}}{a^{4}}
$$

for two semi-spaces and

$$
\frac{F_{s l}(a)}{F_{s l}^{(0)}(a)}=1-4 \frac{\delta_{0}}{a}+\frac{72}{5} \frac{\delta_{0}^{2}}{a^{2}}-\frac{320}{7}\left(1-\frac{\pi^{2}}{210}\right) \frac{\delta_{0}^{3}}{a^{3}}+\frac{400}{3}\left(1-\frac{163 \pi^{2}}{7350}\right) \frac{\delta_{0}^{4}}{a^{4}}
$$

for a sphere (lens) above a semi-space.

In Fig. 3(a) (two semi-spaces) the dashed line 1 represents the results obtained by (26) for $A l$ with $\lambda_{p}=107 \mathrm{~nm}$ (which corresponds to $\omega_{p}=11.5 \mathrm{eV}$ ), and the dashed line 2 - the results obtained by (26) for $A u$ with $\lambda_{p}=136 \mathrm{~nm}$ $\left(\omega_{p}=9 \mathrm{eV}\right)$ [25]. In Fig. 3(b) the dashed lines 1 and 2 represent the perturbation results obtained for $A l$ and $A u$ by (27) for a lens above a semi-space. As is seen from the last column of Table 1, the perturbation results are in good (up to 0.01) agreement with computations for all distances larger than $\lambda_{p}$. Only at $a=0.1 \mu \mathrm{m}$ for $A u$ there are larger deviations because $\lambda_{p 1} \equiv \lambda_{p}^{A u}>0.1 \mu \mathrm{m}$. 


\section{THE CASIMIR FORCE BETWEEN LAYERED SURFACES}

In this Section we consider the influence of the thin outer metallic layers on the Casimir force value. Let the semi-space made of $A l\left(\varepsilon_{2}\right)$ be covered by $A u\left(\varepsilon_{1}\right)$ layers as shown in Fig. 1. For a configuration of a sphere above a plate such covering made of $A u / P d$ was used in experiments [4, \&] with different values of layer thickness $d$. In this case the Casimir force is given by the Eqs. (19), (22), where the quantities $Q_{1,2}(i \xi)$ are expressed by Eqs. (17), (18). The computational results for $\varepsilon_{\alpha}(i \xi)$ are obtained in the previous Section by Eq. (24). Substituting them into (19), (22) and performing a numerical integration in the same way as above one obtains the Casimir force including the effect of covering layers. The computational results for a configuration of two semi-spaces are shown in Fig. 4(a). Here the solid lines represent once more the Casimir force between semi-spaces of pure $A l$ and $A u$ respectively, the dashed and dotted lines are for the case of $A u$ layers of thickness $d=20 \mathrm{~nm}$ and $d=30 \mathrm{~nm}$ covering $A l$. When the layers are present, the space separation $a$ is measured from their outer surfaces according to Eqs. (19), (22). In Fig. 4(b) the analogous results with the same notations are presented for the configuration of a sphere (lens) above a semi-space.

As is seen from Fig. 4, the $A u$ layer of $d=20 \mathrm{~nm}$ thickness significantly decreases the relative Casimir force between $A l$ surfaces. With this layer the force approaches the value for pure $A u$ semi-spaces. For a thicker $A u$ layer of $d=30 \mathrm{~nm}$ thickness the relative Casimir force is scarcely affected by the underlying $A l$. For example, at a space separation $a=300 \mathrm{~nm}$ in the configuration of two semi-spaces we have $F_{s s} / F_{s s}^{(0)}=0.773$ for pure $A l, F_{s s} / F_{s s}^{(0)}=0.727$ for $A l$ with $20 \mathrm{~nm} A u$ layer, $F_{s s} / F_{s s}^{(0)}=0.723$ for $A l$ with $30 \mathrm{~nm} A u$ layer, and $F_{s s} / F_{s s}^{(0)}=0.720$ for pure $A u$. In the same way for the configuration of a sphere above a semi-space the results are: $F_{s l} / F_{s l}^{(0)}=0.817($ pure $A l), 0.780(A l$ with $20 \mathrm{~nm} A u$ layer), 0.776 ( $A l$ with $30 \mathrm{~nm} A u$ layer), 0.774 (pure $A u$ ). Both limiting cases $d \rightarrow \infty$ and $d \rightarrow 0$ were considered and the results are shown to coincide with that of Sec. III.

Let us now discuss the application range of the obtained results for the case of covering layers. First from a theoretical standpoint, the main question concerns the layer thicknesses to which the obtained formulas (19), (22) and the above computations can be applied. In the derivation of Sec. II the spatial dispersion is neglected and, as a consequence, the dielectric permittivities $\varepsilon_{\alpha}$ depend only on $\omega$ not on the wave vector $\boldsymbol{k}$. In other words the field of vacuum oscillations is considered as time-dependent but space homogeneous. Except for the thickness of a skin layer $\delta_{0}$ the main parameters of our problem are the velocity of the electrons on the Fermi surface $v_{F}$, the characteristic frequency of the oscillation field $\omega$, and the mean free path of the electrons $l$. For the considered region of high frequencies (micrometer distances between the test bodies) the following conditions are valid [36]

$$
\frac{v_{F}}{\omega}<\delta_{0} \ll l
$$

Note that the quantity $v_{F} / \omega$ in the left-hand side of Eq. (28) is the distance travelled by an electron during one period of the field, so that the first inequality is equivalent to the assumption of spatial homogeneity of the oscillating field. Usually the corresponding frequencies start from the far infrared part of spectrum which means the space separation $a \sim 100 \mu \mathrm{m}[6]$. The region of high frequencies is restricted by the short-wave optical or near ultraviolet parts of the spectrum which correspond to the surface separations of several hundred nanometers. For smaller distances absorption bands, photoelectric effect and other physical phenomena should be taken into account. For these phenomena, the general Eqs. (19), (22), however, are still valid if one substitutes the experimental tabulated data for the dielectric permittivity along the imaginary axis incorporating all these phenomena.

Now let us include one more physical parameter - the thickness $d$ of the additional, i.e. Au, covering layer. It is evident that Eqs. (19), (22) are applicable only for layers of such thickness that

$$
\frac{v_{F}}{\omega}<d
$$

Otherwise an electron goes out of the thin layer during one period of the oscillating field and the approximation of space homogeneity is not valid. If $d$ is so small that the inequality (29) is violated the spatial dispersion should be taken into account which means that the dielectric permittivity would depend not only on frequency but on a wave vector also: $\varepsilon_{1}=\varepsilon_{1}(\omega, \boldsymbol{k})$. So, if (29) is violated the situation is analogous to the anomalous skin effect where only space dispersion is important and the inequalities below are valid

$$
\delta_{0}(\omega)<\frac{v_{F}}{\omega}, \quad \delta_{0}(\omega)<l
$$

In our case, however, the role of $\delta_{0}$ is played by the layer thickness $d$ (the influence of nonlocality effects on van der Waals force is discussed in 37,38$]$ ). 
From (28), (29) it follows that for pure $A u$ layers $\left(\lambda_{p} \approx 136 \mathrm{~nm}\right)$ the space dispersion can be neglected only if $d \geq(25-30) \mathrm{nm}$. For thinner layers a more general theory taking into account nonlocal effects should be developed to calculate the Casimir force. Thus for such thin layers the bulk tabulated data of the dielectric permittivity depending only on frequency cannot be used (see experimental investigation [39] demonstrating that for $A u$ the bulk values of dielectric constants can only be obtained from films whose thickness is about $30 \mathrm{~nm}$ or more). That is why the dashed lines in Fig. 4 ( $d=20 \mathrm{~nm}$ layers) are subject to corrections due to the influence of spatial dispersion, whereas the solid lines represent the final result. From an experimental standpoint thin layers of order a few nm grown by evaporation or sputtering techniques are highly porous. This is particularly so in the case of sputtered coatings as shown in [40]. The nature of porosity is a function of the material and the underlying substrate. Thus it should be noted that the theory presented here which used the bulk tabulated data for $\varepsilon_{1}$ cannot be applied to calculate the influence of thin covering layers of $d<20 \mathrm{~nm}$ [4,7] and of $d=8 \mathrm{~nm}$ [8,41] on the Casimir force. The measured high transparency of such layers for the characteristic frequencies [4.7] corresponds to a larger change of the force than what follows from the Eqs. (19), (22). This is in agreement with the above qualitative analyses.

The role of spatial dispersion was also neglected in the paper 42 where an attempt was made to describe theoretically the influence of thin metallic covering layers onto the Casimir force in experiments [4,8]. Also the bulk materials properties were used for the $A u / P d$ films. As shown in [43], the resistivity of sputtered $A u / P d$ films even of $60 \mathrm{~nm}$ thickness have been shown to be extremely high of order $2000 \mathrm{ohm} \cdot \mathrm{cm}$. In [42] it was concluded that the maximum possible theoretical values of the force including the covering layers is significantly smaller than the measured ones.

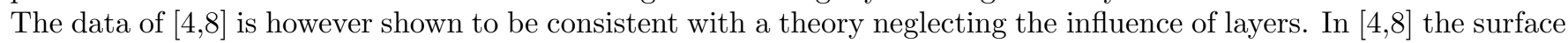
separations are calculated from $\mathrm{Al}$ surfaces. Including the thickness of covering layers reduces the distance between the outer surfaces which is now smaller than the distance between $A l$ surfaces. Thus contrary to 442], the theoretical value of force should increase when the presence of the layers is included. The error made in 42 can be traced to the following. The authors of [42 changed the data of [8] "by shifting all the points to larger separations on $2 h=16 \mathrm{~nm}$ " (where $h=8 \mathrm{~nm}$ is the layer thickness in [8]) instead of shifting to smaller separations by $16 \mathrm{~nm}$ as based on [8]. If the correct shift is done then the theoretical values of the force, including the effect of covering layers, are not smaller than the experimental values. Hence the conclusion in 42 about the probable influence of new hypothetical attractions based on the experiments [4,8] is unsubstantiated.

\section{THE VAN DER WAALS FORCE AND INTERMEDIATE REGION}

As is seen from Figs. 3,4 at room temperature the Casimir force does not follow its ideal field-theoretical expressions (23). For the space separations less than $a=1 \mu \mathrm{m}$ the corrections due to finite conductivity of the metal are rather large (thus, at $a=1 \mu \mathrm{m}$ they are around $7-9 \%$ for a lens above a semi-space, and $10-12 \%$ for two semi-spaces; at $a=0.1 \mu \mathrm{m}$ - around $38-44 \%$ (sl), and $45-52 \%$ (ss)). For $a>1 \mu \mathrm{m}$ the temperature corrections increase very quickly (see Sec. III). Actually, the range presented in Figs. 3,4 is the beginning of a transition with decreasing $a$ from the Casimir force to the van der Waals force. Our aim is to investigate the intermediate region in more detail for smaller $a$ and to find values of $a$ where the pure (non-retarded) van der Waals regime starts. To do this for the case when no additional covering layers are present we numerically evaluate the integrals in Eqs. (20)-22) for $a<100 \mathrm{~nm}$.

The computational results obtained by the same procedures as in Sec. III are presented in Fig. 5(a) for two semispaces and 5(b) for a sphere above a semi-space. In both Figs. the solid line represents the results for aluminum test bodies, and the dashed line for gold ones. The absolute values of the van der Waals force and surface separation $a$ are plotted along the vertical and horizontal axes in a logarithmic scale. The asymptotic expressions in the limit of $a \ll \lambda_{0}$ following from Eqs. (20)-(22) respectively are [11]

$$
F_{s s}^{(0)}(a)=-\frac{H}{6 \pi a^{3}}, \quad F_{s l}^{(0)}(a)=-\frac{H R}{6 a^{2}} .
$$

Here it is important to note that the Hamaker constant $H$ is dependent on the material properties of the boundaries and is a priori unknown. This is in contrast to the ideal Casimir force limit of Eq. (23) (obtained for $a \gg \lambda_{0}$ ) which is material independent and is only a function of $\hbar$ and $c$. Thus it is not reasonable to express the van der Waals force as a ratio relative to Eq. (31). The asymptotic behavior (31) will be used below to determine the value of $H$.

The computations were performed with a step $\Delta a=5 \mathrm{~nm}$ in the interval $10 \mathrm{~nm} \leq a \leq 100 \mathrm{~nm}, \Delta a=1 \mathrm{~nm}$ in the interval $4 \mathrm{~nm} \leq a \leq 10 \mathrm{~nm}, \Delta a=0.2 \mathrm{~nm}$ in the interval $2 \mathrm{~nm} \leq a \leq 4 \mathrm{~nm}$, and $\Delta a=0.1 \mathrm{~nm}$ for $0.5 \mathrm{~nm} \leq a \leq 2 \mathrm{~nm}$. At $a=100 \mathrm{~nm}$ the force values coincide with those in Fig. 3. For $a<0.5 \mathrm{~nm}$ the repulsive exchange forces dominate. As is seen from Fig. 5 for both configurations and two metals under consideration $(A l$ and $A u)$ the range of purely van der Waals force described by Eqs. (31) turn out to be extremely narrow. It extends from $0.5 \mathrm{~nm}$ till $2-4 \mathrm{~nm}$ only. For larger distances the transition from the force-distance dependence $\sim a^{-3}$ to the dependence $\sim a^{-4}$ begins (for 
two semi-spaces) and from the dependence $\sim a^{-2}$ to $\sim a^{-3}$ (for a lens above a semi-space). This conclusion is in a qualitative agreement with the results of [18] where the van der Waals force between a metallic sample and a metallic tip of the atomic force microscope was calculated (our choice of a sphere is formally equivalent to the paraboloidal tip considered in [18). Calculation in 18 was performed by numerical integration of Lifshitz-type equation for the force with the permittivity of a metal given by the plasma model [Eq.(25) with $\gamma=0$ ]. Strictly speaking plasma model is not applicable for $a \ll \lambda_{0}$ (see Sec. III). That is why we have used the optical tabulated data for the complex refractive index in our computations. However, the correct conclusion about the extremely narrow distance range of the purely van der Waals region for metals is obtainable by using the plasma model to represent their dielectric properties. Note that for dielectric test bodies the pure van der Waals regime extends to larger distances. For example in the configuration of two crossed mica cylinders (which is formally equivalent to a sphere above a semi-space) the van der Waals regime extends from $1.4 \mathrm{~nm}$ till $12 \mathrm{~nm}$ as was experimentally shown in 44.

\section{DETERMINATION OF HAMAKER CONSTANTS FOR $A L$ AND $A U$}

The results of the previous Sec. make it possible to determine the values of the Hamaker constant $H$ from Eq. (31) for aluminum and gold. Let us start with the configuration of two semi-spaces. As is seen from the computational results presented in Fig. 5(a) (solid curve) the asymptotic regime for $A l$ extends here from $a=0.5 \mathrm{~nm}$ till $a=4 \mathrm{~nm}$. We use a more narrow interval $0.5 \mathrm{~nm}-2 \mathrm{~nm}$ for the determination of $n$ and $H$. The power index $n$ of the force-distance relation given by the first formula of Eq. (31) is equal to $n=3.02 \pm 0.01$ in the considered interval. To obtain this value the slopes between adjacent points, i.e. $(0.5-0.6) \mathrm{nm},(0.6-0.7) \mathrm{nm}$ etc were calculated and then the average and the standard deviation were found. The corresponding mean value of the Hamaker constant is

$$
H_{s s}^{A l}=(3.67 \pm 0.02) \times 10^{-19} \mathrm{~J} .
$$

Considering the computational results for $A u$ (dashed curve of Fig. 5(a)) we find the asymptotic regime in a more narrow interval $0.5 \mathrm{~nm}-2 \mathrm{~nm}$ with the power index $n=3.04 \pm 0.02$. The mean value of the Hamaker constant turns out to be equal to

$$
H_{s s}^{A u}=(4.49 \pm 0.07) \times 10^{-19} \mathrm{~J} .
$$

For the configuration of a sphere (lens) above a semi-space the results are presented in Fig. 5(b) (solid curve for $A l$ and dashed curve for $A u$ ). In both cases the asymptotic region extends from $a=0.5 \mathrm{~nm}$ till $a=2 \mathrm{~nm}$ only with the mean values of power index in the second formula of Eq. (31) $n=2.04 \pm 0.02(A l)$ and $n=2.08 \pm 0.03(A u)$. The corresponding mean values of the Hamaker constant are

$$
H_{s l}^{A l}=(3.60 \pm 0.06) \times 10^{-19} \mathrm{~J}, \quad H_{s l}^{A u}=(4.31 \pm 0.14) \times 10^{-19} \mathrm{~J} .
$$

It is seen that in the case of $A u$ and a sphere above a semi-space configuration the behavior of the force shows less precise agreement with the second formula of Eq. (31).

The above results obtained for the two configurations independently give the possibility to derive new values of the Hamaker constant for $A l$ and $A u$. Taking into account the value of (32) and the first expression from (34) we get

$$
H^{A l}=(3.6 \pm 0.1) \times 10^{-19} \mathrm{~J} .
$$

The absolute error here was chosen in such a way to cover both permitted intervals in (32) and (34).

For $A u$ the tolerances of the second value from (34) are two times wider than the permitted interval from (33). That is why the most probable final value of the Hamaker constant for gold can be estimated as

$$
H^{A u}=(4.4 \pm 0.2) \times 10^{-19} \mathrm{~J} .
$$

The decreased accuracy than in (35) is explained by the extremely narrow region of pure van der Waals force law for gold. These values of $H$ for gold are compatible with those obtained previously. For example, in [45] values between $(2-4) \times 10^{-19} \mathrm{~J}$ were obtained using different procedures.

\section{CONCLUSIONS AND DISCUSSION}

In the above, general expressions were obtained both for the Casimir energy density and force in the configuration of two plates (semi-spaces) with different separations between them. The case of where the surfaces were covered by the 
thin layers made of the another material was also considered. Additional clarifications of the regularization procedure were given. This is important for obtaining a finite physical value for the energy density. The latter quantity is very important for obtaining the Casimir force for the configuration of a sphere (lens) above a plate (semi-space) which was used in the recent experiments. For this configuration the general expression for the Casimir force with account of layers covering a lens and a semi-space was arrived at by the use of proximity force theorem.

The Casimir force was recalculated between $A l$ and $A u$ test bodies for the configurations of two semi-spaces and a sphere (lens) above a semi-space. The disagreement between the results of [23,24] and [25,26] was resolved in favor of 25,26]. Additionally, computational results were compared with perturbation expansion up to the fourth order in powers of relative penetration depth of electromagnetic zero point oscillations into the metal. The perturbation results are also in agreement with 25,26] and our computations for the space separations larger than a plasma wavelength of the metal under study (not much larger as it to be expected from general considerations). We have performed the first computations of the Casimir force between $A l$ test bodies covered by $A u$ thin layers. The monotonous decrease of the correction factor to the Casimir force was observed with increase of the layer thickness. The qualitative analysis leads to the conclusion that the thickness of the layer should be large enough to allow neglect of the spatial dispersion of the dielectric permittivity and the use of bulk optical tabulated data for the complex refractive index. For the $A u$ layers the minimal allowed thickness for such an approximation was estimated as $d=30 \mathrm{~nm}$ in agreement with the experimental evidence of [39]. For smaller layer thicknesses the bulk optical tabulated data cannot be used. In this case the calculation of the Casimir force would require a direct measurement of the complex refractive index for the particular metal (not only the frequency dependence but also its dependence on the wave vector).

The van der Waals force was calculated between the $A l$ and $A u$ test bodies in configurations of two semi-spaces and a sphere (lens) above a semi-space. The computations were performed starting from the same general expressions as in the case of the Casimir force and using the same numerical procedure and optical tabulated data. The extremely narrow region where the pure non-retarded van der Waals power-law force acts was noted. This region extends from $a=0.5 \mathrm{~nm}$ till $a=(2-4) \mathrm{nm}$ only. For larger distances a wide transition region starts, where the non-retarded van der Waals force described by the Eq. (31) gradually transforms into the retarded van der Waals (Casimir) force from the Eq. (23) when the space separation approaches the value $a=1 \mu \mathrm{m}$. The values of the Casimir force given by the Eq. (31) are never achieved at room temperature (at $a=1 \mu \mathrm{m}$ due to the finite conductivity of the metal while for larger distances the temperature corrections make a strong contribution). Using the asymptotic region of the pure non-retarded van der Waals force the new values of the Hamaker constant for $A l$ and $A u$ were obtained. For $A l$ the reported accuracy corresponds to a relative error of $2.8 \%$, and for $A u$ it is around $4.5 \%$.

The obtained results do not exhaust all the problems connected with the role of finite conductivity of the metal in the precision measurements of the Casimir force. The main problem to be solved is the investigation of corrections to the force due to thin covering layers. This would demand theoretical work on the generalization of the Lifshitz formalism for the case when the spatial dispersion can be important in addition to the frequency dependence. Also the new measurements of the complex refractive index are needed for the layers under consideration. What's more the finite conductivity corrections to the Casimir force should be considered together with the corrections due to the

surface roughness (see, e.g., [7] where the non-additivity of both influential factors is demonstrated) and corrections due to finite temperature. This combined research is necessary for both applied and fundamental applications of the Casimir effect. It has been known that the measurements of the Casimir force give the possibility to obtain strong constraints for the constants of long-range interactions and light elementary particles predicted by the unified gauge theories, supersymmetry and supergravity [6]. Such information is unique and cannot be obtained even by means of the most powerful modern accelerators. In Ref. [35] the constraints for the Yukawa-type hypothetical interactions were strengthened up to 30 times in some distance range on the base of Casimir force measurements of Ref. [3]. The increased precision of the Casimir force in 4 gave the possibility to strengthen constraints up to 140 times on the Yukawa-type interactions at smaller distances [46]. It is highly probable that the new measurements of the Casimir force with increased accuracy will serve as an important alternative source of information about the elementary particles and fundamental interactions.

\section{ACKNOWLEDGMENTS}

G.L.K. and V.M.M. are grateful to the Department of Physics of the Federal University of Paraiba, where this work was partly done, for their hospitality. 
[1] D. Sarid, Scanning Force Microscopy, with Applications to Electric, Magnetic and Atomic Forces (Oxford Univ. Press, N.Y. - Oxford, 1994).

[2] R. Wiesendanger, Scanning Probe Microscopy and Spectroscopy (Cambridge Univ. Press, Cambridge, 1994).

[3] S. K. Lamoreaux, Phys. Rev. Lett. 78, 5 (1997).

[4] U. Mohideen and A. Roy, Phys. Rev. Lett. 81, 4549 (1998).

[5] P. W. Milonni, The Quantum Vacuum (Academic Press, San Diego, 1994).

[6] V. M. Mostepanenko and N. N. Trunov, The Casimir Effect and Its Applications (Clarendon Press, Oxford, 1997).

[7] G. L. Klimchitskaya, A. Roy, U. Mohideen, and V. M. Mostepanenko, Phys. Rev. A 60, 3487 (1999).

[8] A. Roy, C. Y. Lin, and U. Mohideen, Phys. Rev. D 60, R111101 (1999).

[9] E. M. Lifshitz, Sov. Phys. JETP (USA) 2, 73 (1956).

[10] I. E. Dzyaloshinskii, E. M. Lifshitz, and L. P. Pitaevskii, Sov. Phys. Usp. (USA) 4, 153 (1961).

[11] E. M. Lifshitz and L. P. Pitaevskii, Statistical Physics, Part 2 (Pergamon Press, Oxford, 1980).

[12] C. M. Hargreaves, Proc. Kon. Nederl. Acad. Wet. B 68, 231 (1965).

[13] J. Schwinger, L. L. DeRaad, Jr., and K. A. Milton, Ann. Phys. (N.Y.) 115, 1 (1978).

[14] V. M. Mostepanenko and N. N. Trunov, Sov. J. Nucl. Phys. (USA) 42, 812 (1985).

[15] V. B. Bezerra, G. L. Klimchitskaya, and C. Romero, Mod. Phys. Lett. A 12, 2613 (1997).

[16] J. Blocki, J. Randrup, W. J. Swiatecki, and C. F. Tsang, Ann. Phys. (N.Y.) 105, 427 (1977).

[17] V. B. Bezerra, G. L. Klimchitskaya, and V. M. Mostepanenko, e-print quant-ph/9912090.

[18] U. Hartman, Phys. Rev. B 42, 1541 (1990).

[19] U. Hartman, Phys. Rev. B 43, 2404 (1991).

[20] Y. Andersson, D. C. Langreth, and B. I. Lundqvist, Phys. Rev. Lett. 76, 102 (1996).

[21] E. Hult, Y. Andersson, and B. I. Lundqvist, Phys. Rev. Lett. 77, 2029 (1996).

[22] P. H. G. M. van Blokland and J.T.G. Overbeek, J. Chem. Soc. Faraday Trans. 74, 2637 (1978).

[23] S. K. Lamoreaux, Phys. Rev. A 59, R3149 (1999).

[24] S. K. Lamoreaux, Phys. Rev. Lett. 81, 5475 (1998), Erratum.

[25] A. Lambrecht and S. Reynaud, Eur. Phys. J. D 8, 309 (2000).

[26] A. Lambrecht and S. Reynaud, Phys. Rev. Lett., to appear (e-print quant-ph/9912085).

[27] F. Zhou and L. Spruch, Phys. Rev. A 52, 297 (1995).

[28] N. G. van Kampen, B. R. A. Nijboer, and K. Schram, Phys. Lett. A, 26, 307 (1968).

[29] K. Schram, Phys. Lett. A 43, 283 (1973).

[30] H. B. G. Casimir, Proc. Kon. Nederl. Akad. Wet. 51, 793 (1948).

[31] G. L. Klimchitskaya and Yu. V. Pavlov, Int. J. Mod. Phys A 11, 3723 (1996).

[32] V. B. Bezerra, G. L. Klimchitskaya, and C. Romero, Phys. Rev. A 61, 022115 (2000).

[33] M. Bordag, G. L. Klimchitskaya, and V. M. Mostepanenko, Int. J. Mod. Phys. A 10, 2661 (1995).

[34] Handbook of Optical Constants of Solids, edited by E.D. Palik (Academic Press, New York, 1998).

[35] M. Bordag, B. Geyer, G. L. Klimchitskaya, and V. M. Mostepanenko, Phys. Rev. D 58, 075003 (1998).

[36] L. D. Landau and E. M. Lifshitz, Electrodymanics of Continuous Media (Pergamon, Oxford, 1982).

[37] E. I. Kats, Sov. Phys. JETP (USA) 46, 109 (1977).

[38] G. Barton, Rep. on Progr. in Phys. 42, 963 (1979).

[39] P. B. Johnson and R. W. Christy, Phys. Rev. B 6, 4370 (1972).

[40] I. Stokroos, D. Kalicharan, J. J. L. Van der Want, and W. L. Jongebloed, J. of Microscopy 189, 79 (1997).

[41] A. Roy and U. Mohideen, Phys. Rev. Lett. 82, 4380 (1999).

[42] V. B. Svetovoy and M. V. Lokhanin, e-print quant-ph/0001010.

[43] J. R. Kirtley, S. Washburn, and M. J. Brady, Phys. Rev. Lett. 60, 1546 (1988).

[44] J. N. Israelachvili and D. Tabor, Proc. Roy. Soc. Lond. A 331, 19 (1972).

[45] V. A. Parsegian, G. H. Weiss, J. Colloid and Interface Sci. 81, 285 (1981).

[46] M. Bordag, B. Geyer, G. L. Klimchitskaya, and V. M. Mostepanenko, Phys. Rev. D 60, 055004 (1999). 


\section{List of captions}

FIG.1. The configuration of two semi-spaces with a dielectric permittivity $\varepsilon_{2}(\omega)$ covered by layers of thickness $d$ with a permittivity $\varepsilon_{1}(\omega)$. The space separation between the layers is $a$.

FIG.2. The dielectric permittivity as a function of imaginary frequency for $A l$ (solid line) and $A u$ (dashed line).

FIG.3. The correction factor to the Casimir force due to finite conductivity of the metal as a function of the surface separation. The solid line 1 and 2 represents the computational results for $A l$ and $A u$ respectively in the configuration of two semi-spaces (a) and for a sphere (lens) above a semi-space (b). The dashed lines 1 and 2 represent the perturbation correction factor up to the 4 th order for $A l$, and $A u$ respectively.

FIG.4. The correction factor to the Casimir force due to finite conductivity of the metal as a function of the surface separation for $A l$ test bodies covered by thin layers of $A u$. The dashed lines represent the results for a layer thickness $d=20 \mathrm{~nm}$ and the dotted lines for $d=30 \mathrm{~nm}$. The case of the configuration of two semi-spaces is shown in (a) and for a sphere (lens) above a semi-space is shown in (b). The solid lines represent the results for pure $A l$ and $A u$ test bodies respectively.

FIG.5. The absolute value of the van der Waals force as a function of surface separation is shown on a logarithmic scale. The solid lines represent the results for $A l$ and the dashed lines represent the case of $A u$. The configuration of two semi-spaces is shown in (a) and that for a sphere (lens) above a semi-space is shown in (b). 
Fig. 1

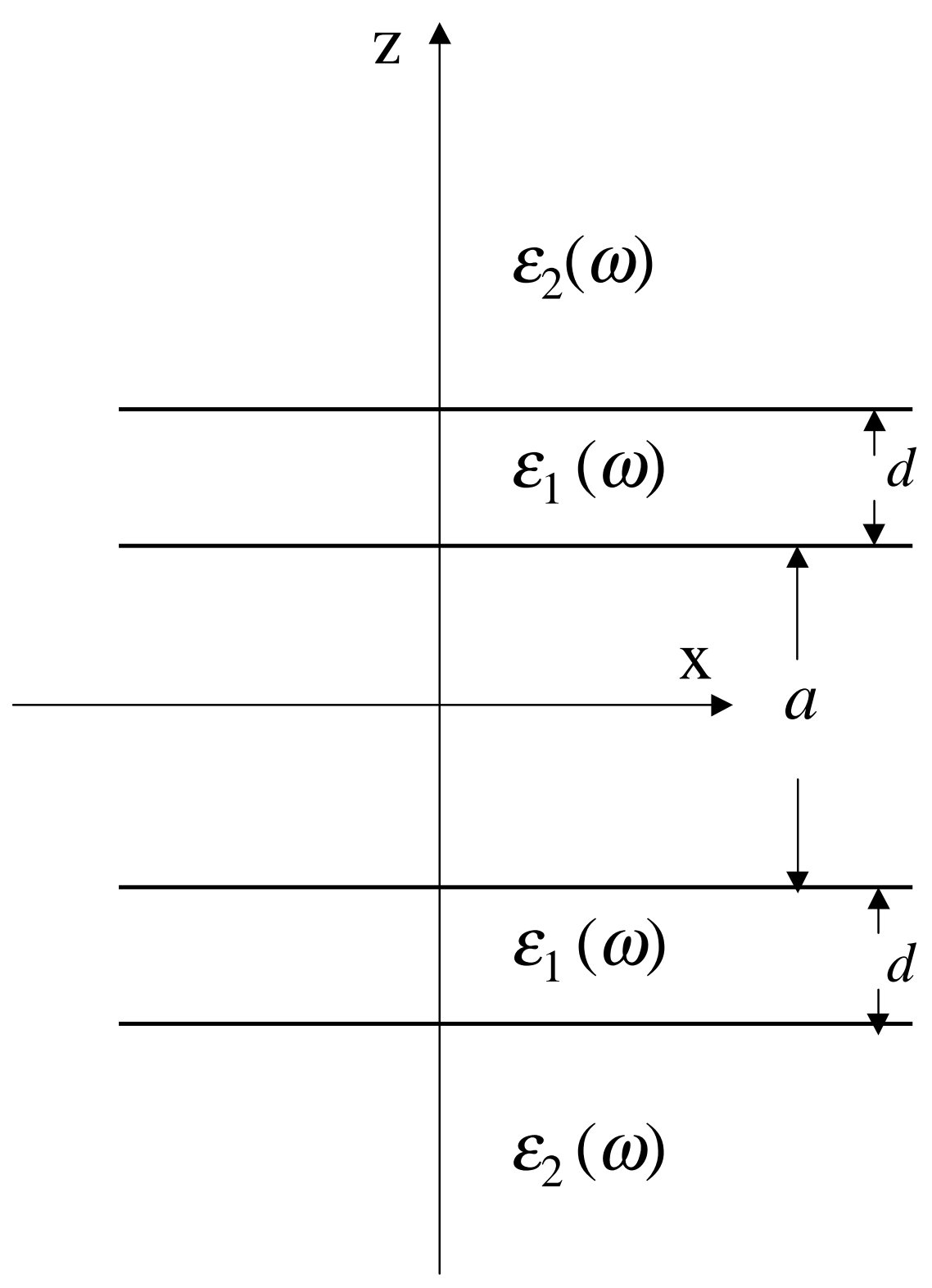


Fig. 2

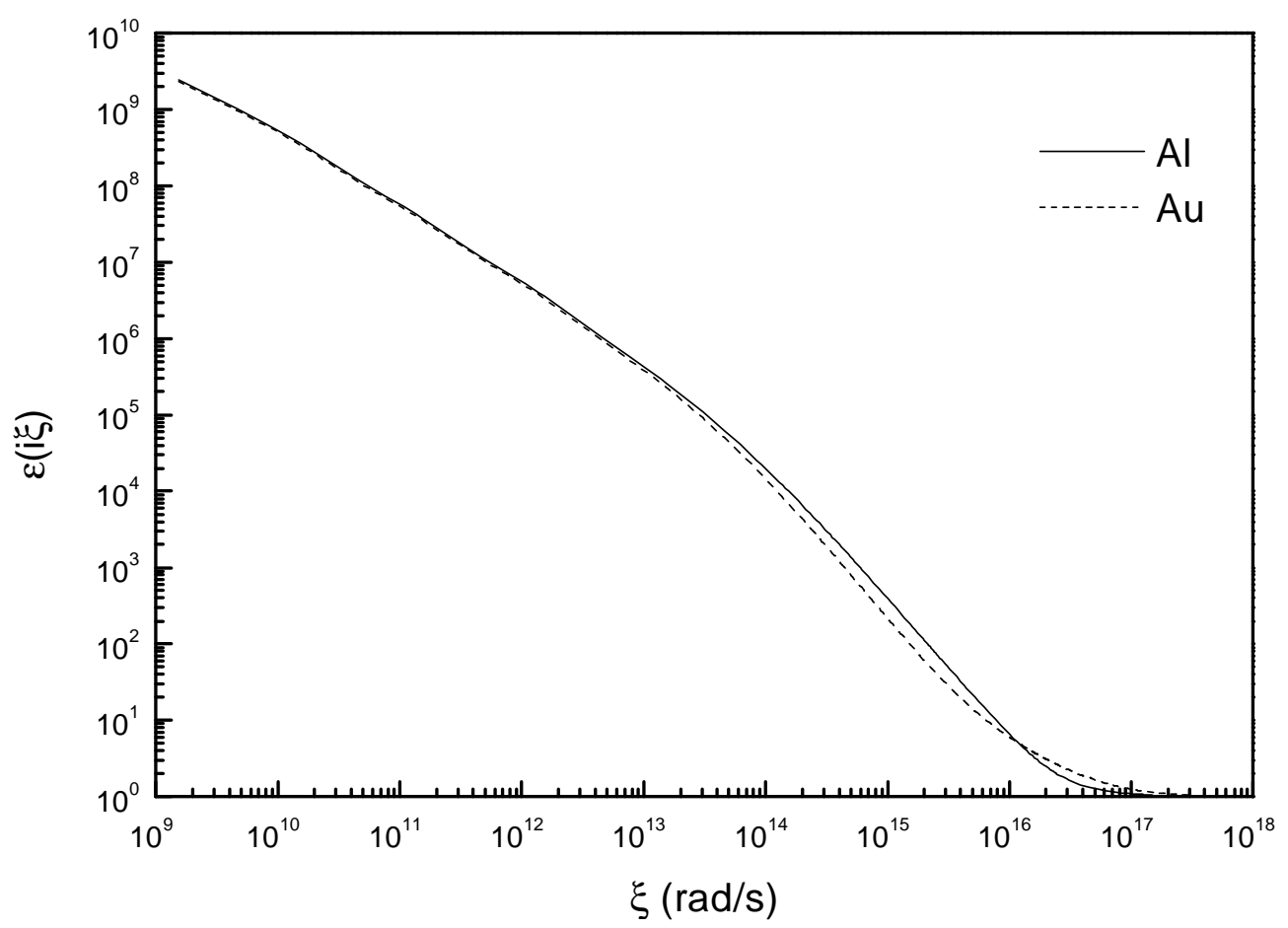


Fig 3(a)

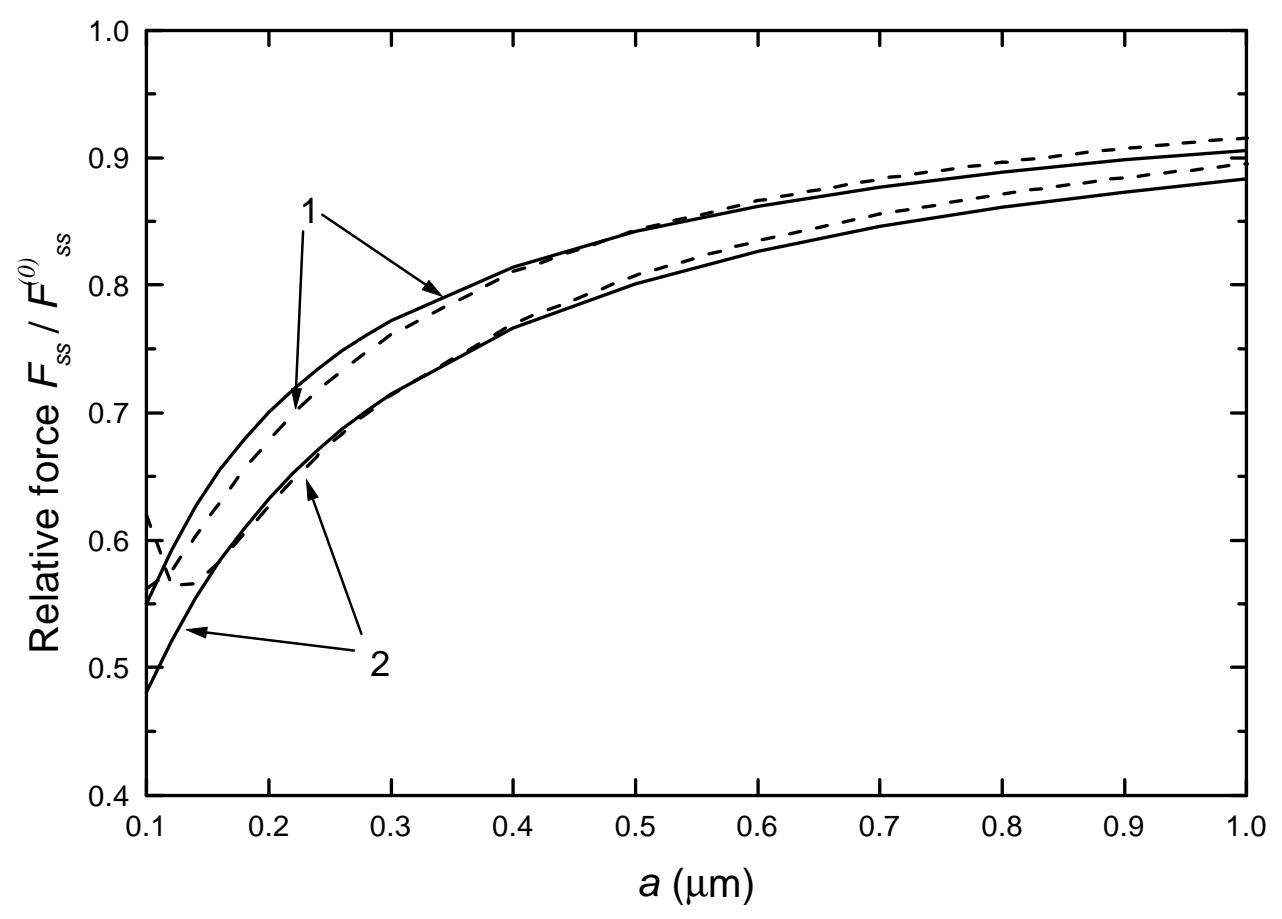


Fig. 3(b)

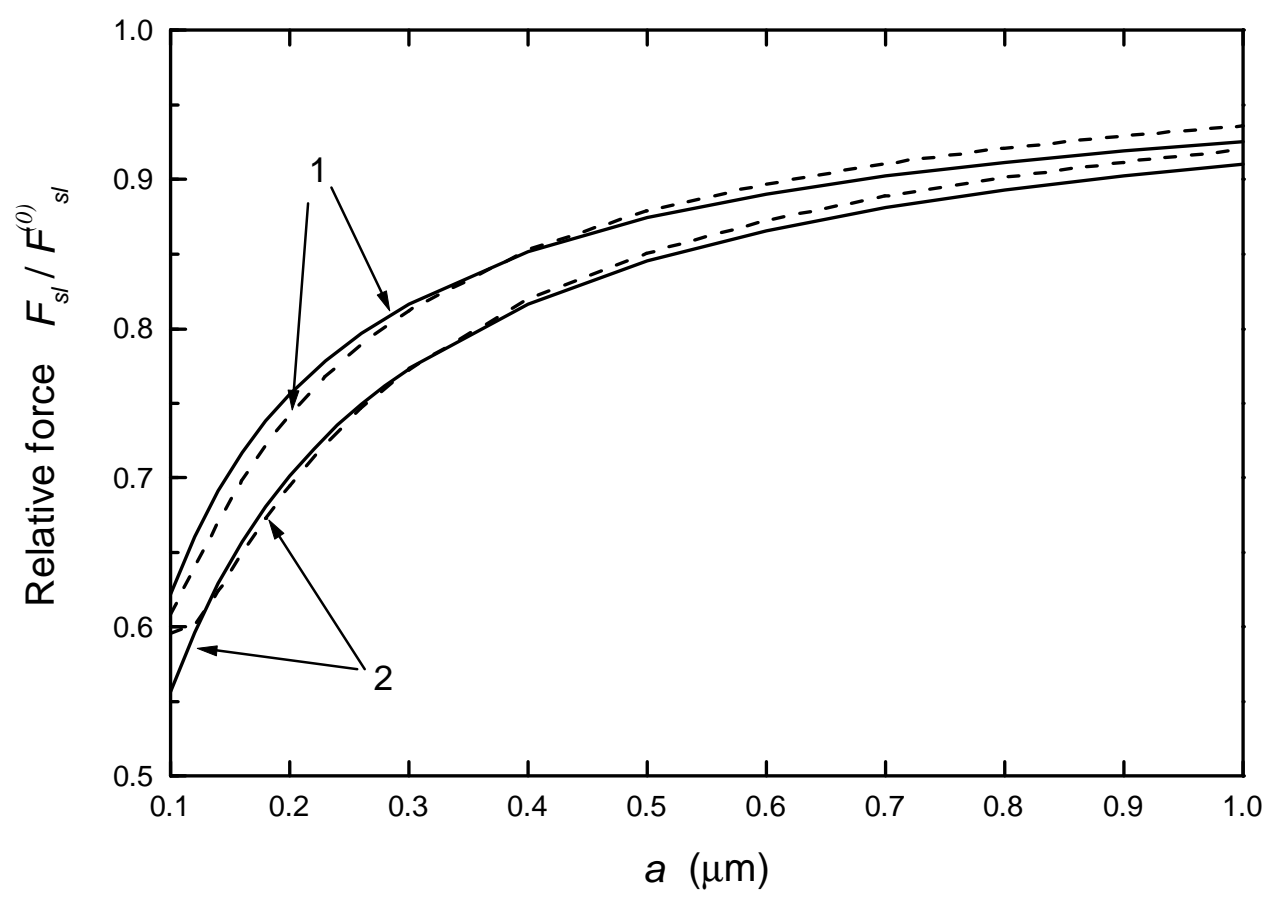


Fig 4(a)

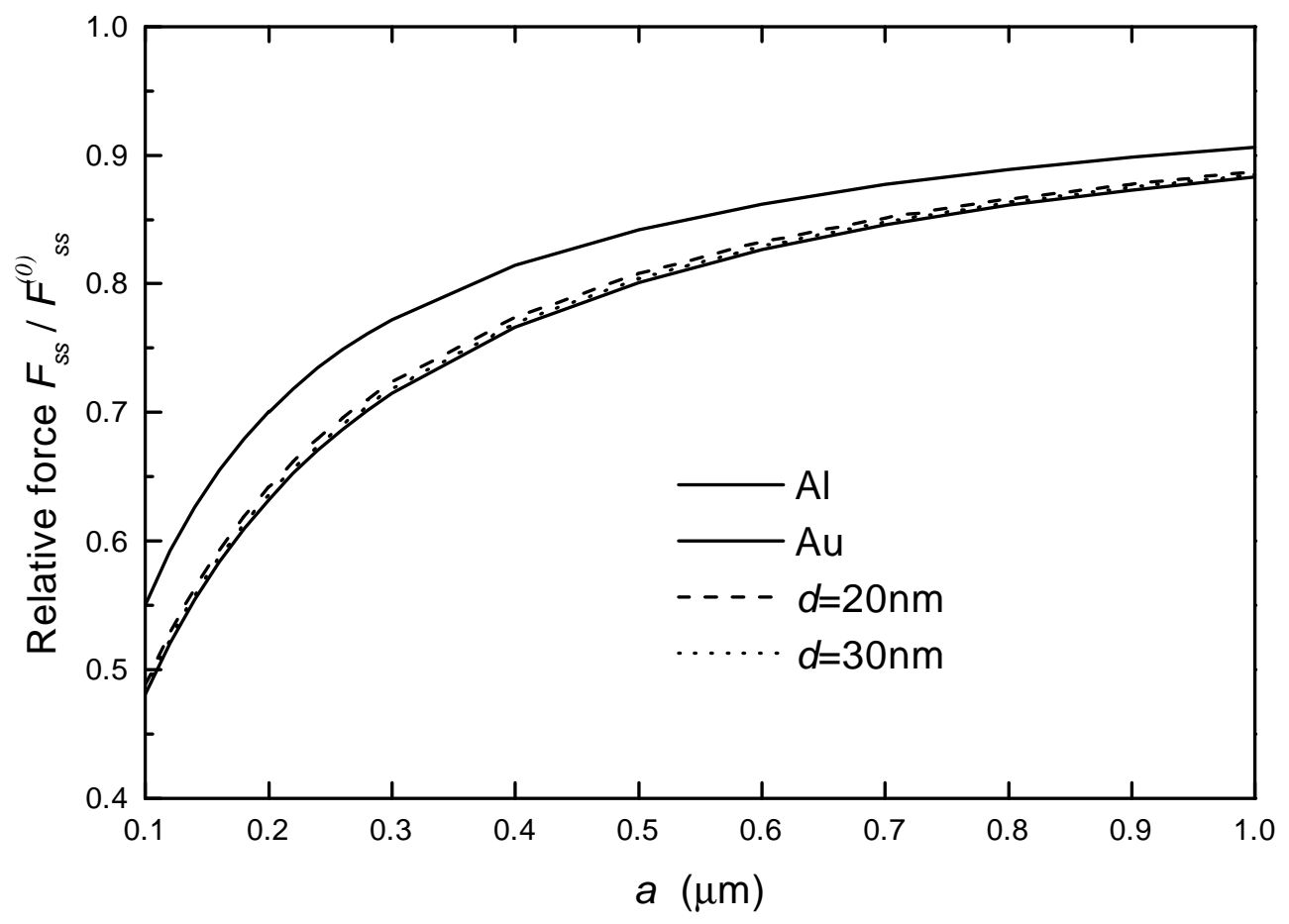


Fig 4(b)

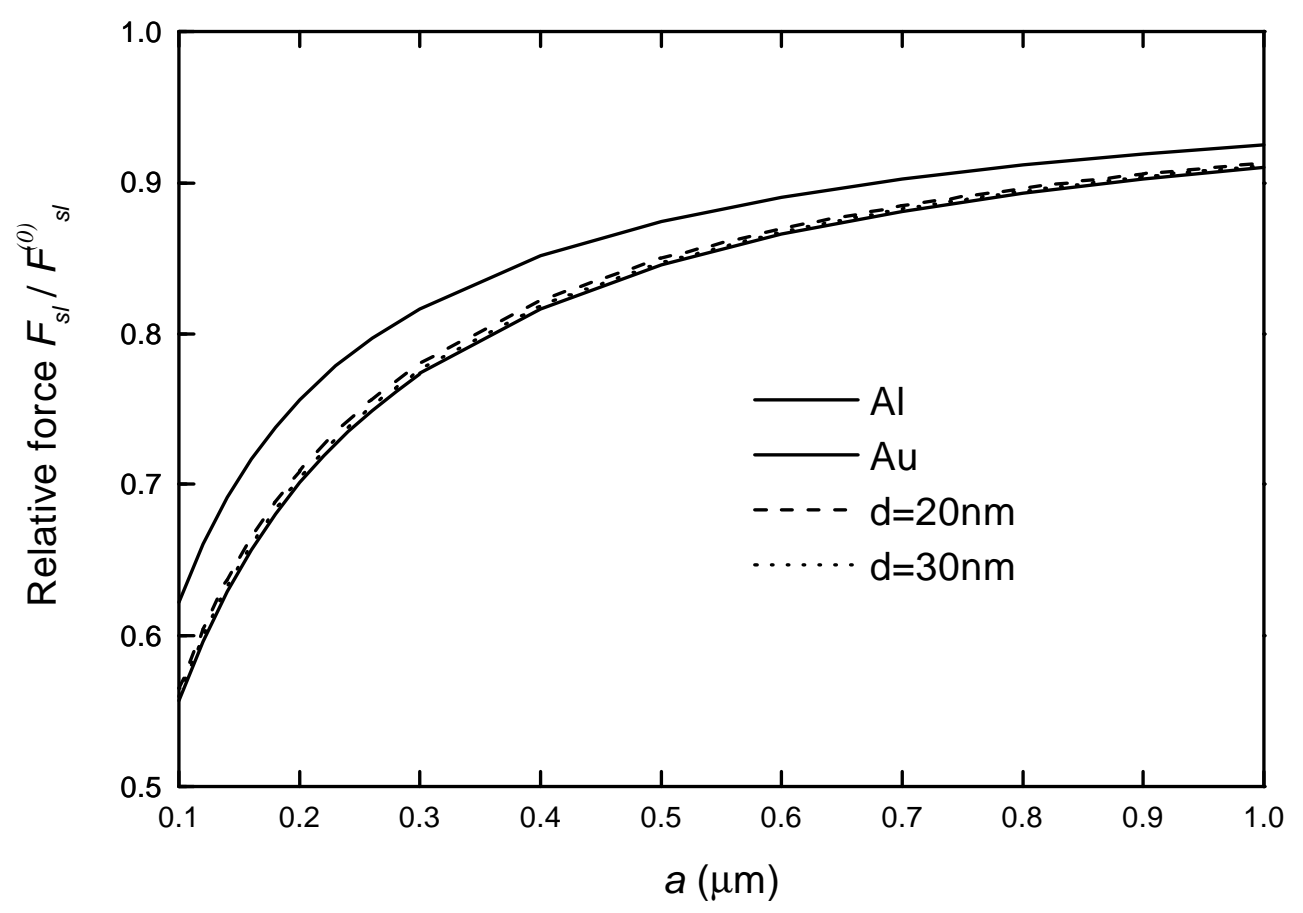


Fig. 5(a)

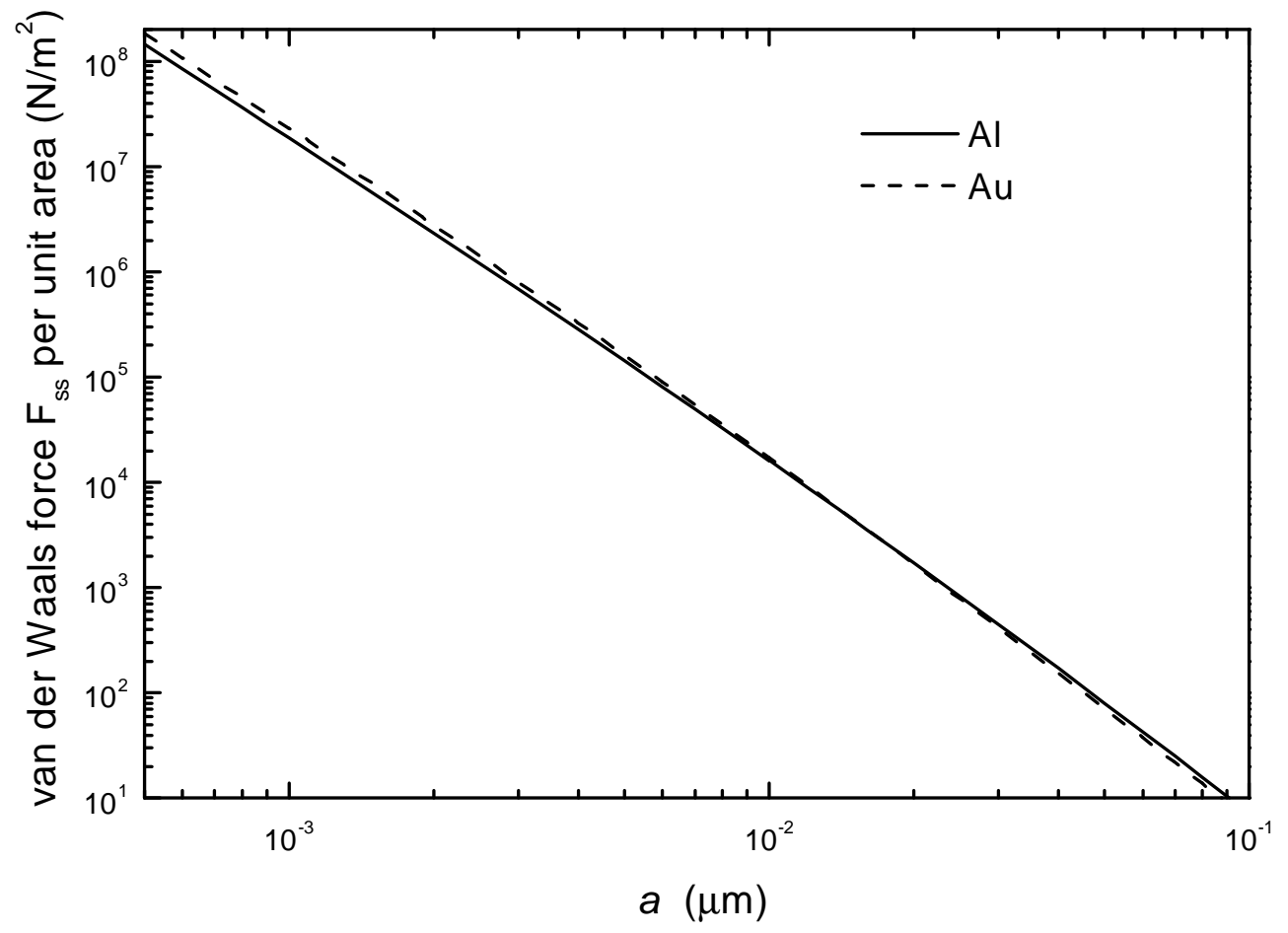


Fig. 5(b)

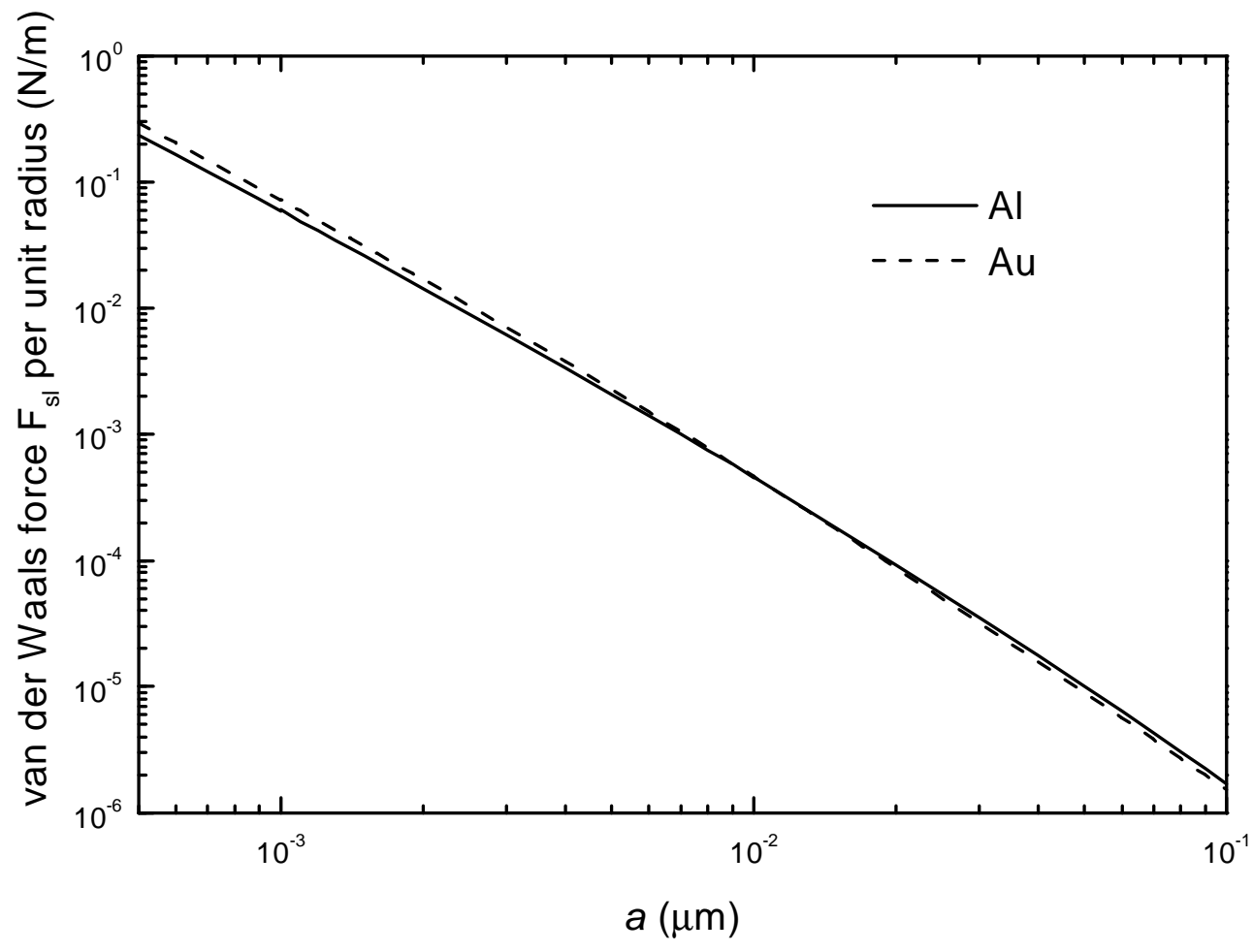

